\title{
ANSIEDAD, DEPRESIÓN Y ACTIVIDAD AUTONÓMICA EN ESTUDIANTES DE ENFERMERÍA, EN EL ESTADO dE GuANAJUATO, MÉXICO
}

\author{
Gerardo Ruvalcaba Palacios, Andrea Galván Guerra, Grever, María Ávila Sansores \\ y Patricia Isolina del Socorro Gómez Aguilar \\ Universidad de Guanajuato, Universidad Autónoma de Yucatán \\ México
}

\begin{abstract}
RESUMEN
Nuestro objetivo fue estudiar las relaciones entre actividad autonómica, ansiedad y depresión de estudiantes de enfermería, siguiendo un diseño correlacional y un muestreo intencional; se aplicaron los inventarios de ansiedad y depresión de Beck, así como el perfil psicofisiológico del estrés. Fueron incluidas 133 personas de primero, tercero y quinto semestre de una universidad de Guanajuato, México. El índice promedio de ansiedad fue de 23.17 (nivel moderado). La media de depresión fue de 7.9 (nivel mínimo). No se encontraron relaciones significativas entre ansiedad y depresión $\left(r_{s}=0.077, p=0.380\right)$, ni entre estas emociones y el nivel de actividad autonómica $(p=0.05)$. En el perfil psicofisiológico se encontraron correlaciones entre los índices obtenidos en la primera condición y los de la última $\left(r_{s}=0.844, p<0.05\right)$. La ansiedad y la depresión son muy prevalentes, pero son necesarios más estudios para clarificar las relaciones que hay entre ellos y la actividad autonómica de estudiantes universitarios.
\end{abstract}

Palabras Clave:

ansiedad, depresión, actividad autonómica, estudiantes universitarios, estrés académico.

\section{ANXIETY, DEPRESSION AND AUTONOMIC ACTIVITY IN NURSING STUDENTS, in the state of Guanajuato, Mexico}

\begin{abstract}
Our aim was to study the relationships between autonomic activity, anxiety and depression levels among nursing students. It was used a correlational design and an intentional sampling, so the anxiety and depression inventories was applied, as well as the psychophysiological stress profile. It was included 133 students of first, third and fifth semester of an university located in Guanajuato, Mexico. The anxiety average score was 23.17 (moderate level of anxiety). The average score of depression was 7.9 (It means a minimal level of depression). It was no founded significant relationships between anxiety and depression row scores $\left(r_{s}=0.077, p\right.$ $=0.380)$, no found neither, relationships between those levels and autonomic activity $(p=0.005)$.

In the psychological profile, it was founded correlations between scores of autonomic activity obtained in the first activity and those obtained in the final condition $\left(r_{s}=0.844, p<0.05\right)$. Anxiety and depression are highly prevalent, but it is necessary more research to clarify the relationships underlying between those and autonomic activity of university students.
\end{abstract}

Keywords:

anxiety, depression, autonomic activity, university students, academic stress.

Bitácora del ARtículo:

| Recibido: 9 de Mayo de 2019 | Aceptado: 1 de Octubre de 2019 | Publicado en línea: Enero-Junio de 2020 | 


\title{
ANSIEDAD, DEPRESIÓN Y ACTIVIDAD AUTONÓMICA EN ESTUDIANTES DE ENFERMERÍA, EN EL ESTADO DE GUANAJUATO, MÉXICO
}

\author{
Gerardo Ruvalcaba Palacios, Andrea Galván Guerra, Grever María Ávila Sansores y Patricia Isolina \\ del Socorro Gómez Aguilar \\ Universidad de Guanajuato, Universidad Autónoma de Yucatán \\ México
}

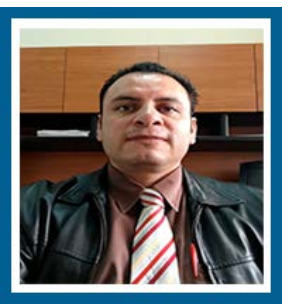

Gerardo Ruvalcaba Palacios

Universidad de Guanajuato

Correo:drruvalcaba@gmail.com

Doctor en Psicología de la Salud UNAM (2006-2011). Miembro del Sistema Nacional de Investigadores (SNI). con la categoría Investigador I (2017-2019). Profesor con reconocimiento al perfil deseable (desde julio 2012).

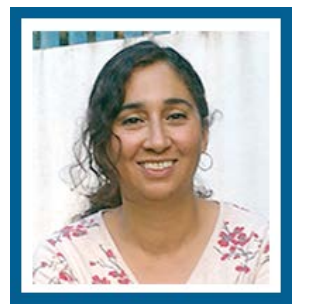

Andrea Galván Guerra Universidad de Guanajuato

Correo: galvan.andrea@gmail.com

Licenciada en Psicología por la Universidad Autónoma de Aguascalientes (UAA). Maestra en Gerontología Social por el Centro Panamericano de Estudios Superiores (CEPES). Estudiante del Doctorado en Psicología en la Universidad Autónoma de Aguascalientes (UAA).

\section{CONTRIBUCIÓN DE LOS AUTORES}

Gerardo Ruvalcaba Palacios: concibió y desarrolló el proyecto. Escribió introducción, resultados, conclusiones y discusión. Aplicó análisis de datos. Leyó todo el manuscrito para su coherencia, buscó la revista para publicar | Andrea Galván Guerra: concibió y desarrolló el proyecto. Aplicó análisis de datos. Escribió introducción, método, resultados, conclusiones y discusión. Leyó todo el manuscrito para coherencia | Grever Ma. Ávila Sansores: escribió conclusiones y discusión. Aplicó entrevistas para el perfil psicofisiológico capturó bases de datos e hizo análisis preliminares | Patricia Isolina del Socorro Gómez Aguilar: Escribió resultados, aplicó encuestas, entrevistas y escribió discusión

\section{AGRADECIMIENTOS}

Se agradece a la Dirección de Apoyo a la Investigación y el Posgrado (DAIP) de la Universidad de Guanajuato por el apoyo brindado a través del proyecto de investigación 328/2014 "Terapia asistida por animales en la demencia de ancianos institucionalizados ¿Cuál es el rol de la actividad autonómica?”

\section{DATOS DE Filiación de los Autores}

Gerardo Ruvalcaba Palacios: Universidad de Guanajuato | Andrea Galván Guerra: Universidad de Guanajuato | Grever Ma. Ávila Sansores: Universidad de Guanajuato | Patricia Isolina del Socorro Gómez Aguilar: Universidad Autónoma de Yucatán

Copyright: (c) 2020 Ruvalcaba-Palacios, G., Galván-Guerra, A., Ávila-Sansores, G. M. \& Gómez-Aguilar, S.

Este es un artículo de acceso abierto distribuido bajo los términos de la licencia Creative Commons Reconocimiento-NoComercial 4.0 Internacional, por lo que su contenido gráfico y escrito se puede compartir, copiar y redistribuir total o parcialmente sin necesidad de permiso expreso de sus autoras con la única condición de que no se puede usar con fines directamente comerciales y los términos legales de cualquier trabajo derivado deben ser los mismos que se expresan en la presente declaración. La única condición es que se cite la fuente con referencia a la Revista Digital Internacional de Psicología y Ciencia Social y a sus autoras. 


\section{TABLA DE CONTENIDO}

MÉTOdO

Participantes, 223

Materiales, 223

Procedimiento, 224

Mediciones, 224

Análisis estadístico, 225

\section{RESULTADOS}

Muestra, 225

Participantes, 225

Estado emocional, 226

Actividad autonómica, 226

Análisis multivariados, regresiones, correlaciones, 228

\section{Discusión}


$\mathbf{L}$ os estados patológicos de tristeza y temor son los sufrimientos más comunes y severos que aquejan a la humanidad, de tal forma que, tanto la depresión como la ansiedad, son considerados un problema significativo de salud (Beiter, et al., 2015; Organización Panamericana de la Salud [OPS], 2017).

Actualmente su prevalencia es tan alta que se cree podrían afectar, en mayor o menor grado, a una gran parte de las personas en edad adulta. En México, la depresión y la ansiedad son también los principales padecimientos psiquiátricos, en una proporción de alrededor de un hombre por cada dos mujeres (Medina-Mora et al., 2009). De acuerdo con las estimaciones sanitarias mundiales, en 2015 se estimaba que el trastorno depresivo afectaba a $4.2 \%$ de la población general y el trastorno de ansiedad a $3.6 \%$. Debido a ello se les ha considerado como padecimientos mentales muy graves que reflejan una pérdida de salud y del funcionamiento general, con un índice de años de vida ajustados por discapacidad (AVAD) de $8.6 \%$ para la depresión y de $4 \%$ para la ansiedad (OPS, 2017).

Desafortunadamente los efectos perniciosos de dichas enfermedades no se limitan al sufrimiento que ocasionan de modo directo a las personas que los padecen, sino que se extienden a familiares y amigos porque los síntomas ocasionan alteraciones en las relaciones sociales e incluso en la productividad, además de que promueven en los pacientes el involucramiento en conductas poco saludables o que ponen en riesgo la salud. Por ejemplo, hasta $37 \%$ de las personas con ansiedad, y hasta $50 \%$ de los afectados por depresión, también presentan patrones de abuso de drogas (Medina-Mora et al., 2009).

En el ámbito laboral se ha documentado que quienes padecen ansiedad o depresión presentan también mayores niveles de discapacidad; incluso que quienes tienen algún tipo de condición crónica como diabetes, hipertensión, artritis, cefaleas o dolores de espalda (Ibrahim, Kelly, Adams y Glazebrook, 2013). En nuestro país las personas que padecen algún trastorno crónico solicitaron en promedio 6.9 días de incapacidad laboral al año, los afectados por depresión solicitaron en promedio hasta 25.5 días, y los que padecen cualquier condición asociada a los trastornos de ansiedad (como ataques de pánico, fobia social, ansiedad generalizada o estrés pos- traumático) hasta alrededor de 17 días de ausencia laboral en promedio por año (Medina-Mora et al., 2009).

Además, en México estos trastornos se presentan a edades cada vez más tempranas y con una incidencia creciente entre la población general. Incluso se ha detectado un efecto de cohorte en el cual los jóvenes tienen más riesgo de padecer estos síndromes que las generaciones anteriores. Este fenómeno se ha observado de modo significativo entre quienes están en edad universitaria (en el rango de 18 a 26 años de edad), pues en ellos se ha documentado una tendencia hasta 2.5 veces mayor de padecer ansiedad y hasta 6.3 veces más de sufrir cualquier trastorno afectivo que las personas mayores (Medina-Mora et al., 2009).

El mismo escenario epidemiológico se observa también en otros países, como en Estados Unidos, donde Beiter et al. (2015) documentaron que en 2014 hasta siete de cada 10 jóvenes universitarios experimentaron diariamente síntomas de estos trastornos, y uno de cada 10 fue diagnosticado con alguno de ellos. Los autores indican que la demanda de servicios psicológicos o de consejería estudiantil por esta causa también se había incrementado entre 175 y $235 \%$ en ese mismo año.

De acuerdo con Yusoff et al. (2013), estos incrementos en la prevalencia de ansiedad y depresión entre los universitarios se relacionan de modo directo con la elevada exigencia que caracteriza a los sistemas actuales de educación superior. Y no se habla sólo de las cargas académicas, las evaluaciones, la cantidad de material que es necesario aprender, o la distribución del tiempo; también se ha documentado que la necesidad de obtener buenas calificaciones es una fuente importante de estrés, ansiedad y depresión para los estudiantes, sobre todo porque esto les permitirá acceder luego a oportunidades de becas y otros apoyos (Beiter et al., 2015).

Otros factores de riesgo significativos están asociados con el desarrollo de las actividades extracurriculares que la formación universitaria exige, además de la presión familiar y social para ser exitoso y lograr destacar después de egresar (Regehr, Glancy y Pitts, 2013). Respecto a los factores de riesgo individuales, Yussoff et al. (2013) identificaron que la baja autoestima, los sentimientos de inferioridad, la falta de reconocimiento social o en el desempeño escolar, los malos hábitos de sueño, así como el provenir de familias con dificultades económicas son también fuentes significativas de ansiedad y depresión.

Sin embargo, y aunque hoy los niveles de ansiedad y depresión de los estudiantes son muy elevados, tal vez quienes se forman en el área de la salud son más proclives a desarrollar trastornos emocionales (Agudelo, Casadiegos y Sánchez, 2008). 
De las diferentes áreas de la salud, los estudiantes de la carrera de enfermería se han identificado por ser uno de los grupos que más padecen estos trastornos ( $\mathrm{Ji}$ ménez, Martínez y Vacas, 2010); incluso algunos autores han identificado que el nivel de estrés de los estudiantes de esta carrera es hasta $68 \%$ mayor que el que presentan los de medicina (Jones y Johnston, 1997; como se citó en Consolo, Fusner y Staib, 2008). Otros estudios indican que hasta $30 \%$ de los estudiantes de enfermería padecen depresión, y que de este índice hasta $67 \%$ presenta también ansiedad (Song y Lindquist, 2014).

Como sucede con estudiantes de otras carreras, parece que la exigencia académica es suficiente para desarrollar altos niveles de estrés entre los que estudian enfermería (Chan, Creedy, Chua y Lim, 2011); sin embargo, a diferencia de otros programas, estos estudiantes enfrentan también la necesidad de equilibrar dichas exigencias con las responsabilidades clínicas, factor que se ha asociado con el desarrollo de ansiedad y depresión (Consolo et al., 2008), sobre todo porque aún en etapas tempranas de su formación reciben la responsabilidad de procurar el bienestar, e incluso la vida, de sus pacientes (Watson et al., 2013).

Además de las responsabilidades clínicas que tienen asignadas, en ocasiones el ambiente hospitalario es hostil y no ofrece la seguridad y pertenencia que los estudiantes necesitan para aprender en un ambiente relajado y propicio (Grobecker, 2016), pues, por ejemplo, no sólo tienen que lidiar con los superiores de su disciplina, sino que tienen que interactuar con otros grupos de profesionales y su respectiva organización jerárquica, además de enfrentar situaciones difíciles con los pacientes y sus familiares, e inevitablemente el sufrimiento que supone la agonía y muerte de algunas de las personas a su cuidado (Jiménez et al., 2010; Watson et al., 2013).

De esta manera se ha reconocido que con frecuencia los estudiantes de enfermería padecen altos niveles de estrés, lo que deriva en el desarrollo de trastornos de ansiedad y depresión, así como en detrimento de su capacidad para aprender y desarrollar una práctica clínica adecuada (Jiménez et al., 2010; Song y Lindquist, 2014).

Una relación causal entre niveles elevados de estrés y el desarrollo de trastornos de ansiedad y depresión ya ha sido documentada (Beiter et al., 2015). Por ejemplo, se ha determinado que la exposición repetida a sucesos estresantes durante las diferentes etapas del crecimiento ocasiona deterioro en el proceso de regulación emocional, lo que se relaciona con el desarrollo de ansiedad y depresión, así como de otros trastornos psiquiátricos (Abravanel y Sinha, 2015). Es probable que los estados crónicos de activación simpática condicionan el establecimiento de respuestas adaptativas caracterizadas por estados de ansiedad y depresión (Streeter, Gerbarg, Saper, Ciraulo y Beown, 2012).

En este sentido, algunos autores (Dieleman et al., 2015; Sapolsky, 2003; Streeter et al., 2012) han documentado que, en los estados crónicos de estrés, la presencia de niveles elevados de glucocorticoides afecta de manera notable estructuras específicas del cerebro relacionadas con la integración de las respuestas de lucha-huida del organismo; en especial la amígdala, encargada de procesar los estímulos que desencadenan reacciones de miedo. Como resultado, erróneamente el organismo interpreta el contexto como amenazante, lo que deriva en un estado de alerta generalizada. Este estado de ansiedad constante y la liberación constante de hormonas precursoras del estrés afectan la producción de dopamina. El descenso en los niveles de dicho neurotransmisor condiciona que la ansiedad intensa derive en estados de anhedonia, una falta de capacidad para disfrutar la vida y, en general, el alejamiento emocional que caracteriza a la depresión. Una revisión de estos postulados puede analizarse en Kemp y Felmingham (2008).

Así, de manera general puede decirse que una característica común a estos trastornos emocionales es la presencia de una elevada activación simpática (Dieleman et al., 2015; Van Reedt Dortland et al., 2013), por lo que se puede esperar que los estudiantes que tienen elevados niveles de actividad simpática, también muestren altos niveles de ansiedad y depresión.

Para indagar las respuestas de estrés, en la investigación psicofisiológica con frecuencia se ha utilizado la conductancia galvánica de la piel (CGP) porque es un indicador confiable de actividad simpática (Treister, Kliger, Zuckerman, Aryeh y Eisenberg, 2012). De igual modo, este sistema psicofisiológico es uno de los más utilizados en psiquiatría, psicología, psicofisiología y otras áreas, como lo demuestra la gran cantidad de artículos científicos que se han publicado (Ruvalcaba \& Galván, 2017). Sin embargo, en México la señal ha sido poco utilizada y por lo mismo son pocos los estudios que al respecto se han elaborado.

De igual manera y hasta donde se sabe, en México no hay estudios que documenten las relaciones entre el estado afectivo de estudiantes de enfermería (medido con los niveles de ansiedad y depresión) y la actividad autonómica que presentan.

Por tanto, este estudio se efectuó con el objetivo de estudiar las relaciones entre el nivel de actividad autonómica simpática y los niveles de ansiedad y depresión en una muestra de estudiantes de enfermería de una universidad en el estado de Guanajuato, México. 
Como objetivos específicos se quería conocer los niveles de ansiedad, depresión y actividad autonómica por sexo y por grado escolar de los participantes; los cambios en la actividad autonómica que los participantes mostraban en cada una de las condiciones que conformaban el perfil psicofisiológico del estrés, y saber si las variables sociodemográficas de los estudiantes de enfermería se relacionaban con los niveles de ansiedad, depresión o de actividad autonómica.

El conocimiento adquirido ayudaría a justificar el desarrollo de programas de intervención dirigidos a la modulación consciente de la actividad autónoma asociada a la presencia de estas alteraciones emocionales.

\section{Método}

\section{Participantes}

Se utilizó un estudio correlacional y mediante un muestreo intencional se incluyeron a todos los alumnos regulares inscritos en el semestre agosto-diciembre 2014 en el programa educativo de enfermería de una universidad de Guanajuato, México. Se consideraron alumnos regulares a los que no adeudaran materias, que no tuvieran menos o más carga académica que sus compañeros y/o que no estuvieran de intercambio en alguna institución educativa o de investigación.

Se excluyeron los inventarios y registros incompletos o llenados de manera inadecuada. También se descartaron los participantes que no hicieron la evaluación psicofisiológica.

\section{Materiales}

\section{Inventario de ansiedad de Beck}

Estandarizado para población mexicana (Robles, Varela, Jurado y Páez, 2001); consta de 21 reactivos que indican diferentes síntomas físicos y cognitivos de ansiedad. El participante los califica de acuerdo con una escala tipo Likert con valencias de cero ("poco o nada") a tres ("severamente"), según los haya experimentado durante la semana previa a su Ilenado. Este inventario con frecuencia se utiliza en escenarios clínicos y no clínicos para evaluar sintomatomas de ansiedad tanto en adolescentes como en adultos, mostrando una alta consistencia interna (alfa > 0.9) y una validez convergente superior a 0.50 (Galindo, Rojas, Meneses, Aguilar, Álvarez y Alvarado, 2015).

\section{Inventario de depresión de Beck}

Estandarizado para población mexicana (Jurado et al., 1998) consta de 21 grupos de cuatro oraciones, las cuales están numeradas de 0 a 3; el orden indica la severidad de los diferentes síntomas de depresión. Es un instrumento que comúnmente se usa en la investigación de depresión; tiene una sensibilidad de 0.92 y una especifidad de 0.59, además de una buena consistencia interna con un alfa $=0.92$, lo que hace de este un instrumento que se usa de manera constate para propósitos clínicos y científicos con poblaciones adolescentes y adultas (Beltrán, Freyre y Hernández-Guzmán, 2012).

\section{Actividad autonómica}

Se utilizó un perfil psicofisiológico del estrés, el cual es un procedimiento que permite registrar el nivel de reactividad autonómica ante diferentes tipos de estrés, así como la capacidad del organismo para volver a sus niveles basales después de dichos tipos de eventos (Ruvalcaba y Domínguez, 2008).

Está conformado por tres condiciones: 1) estresor cognitivo (EC), o Stroop, en el cual de manera rápida se presentan al participante diapositivas que contienen el nombre de diferentes colores, escritos con un color distinto al del nombre (por ejemplo "azul" escrito con tinta roja); el participante debe mencionar el color de la tinta, no el del nombre del color (en el ejemplo debe mencionar "rojo", ante la visualización del nombre "azul"); 2) estresor emocional (EE); se solicita al participante que recuerde lo más vívidamente posible un suceso reciente de ansiedad o depresión y que lo reviva "como si lo estuviera experimentando en este momento", y 3) respuesta natural de relajación (RNR); se pide al participante que se relaje de modo que sepa o crea pueda hacerlo.

Entre cada condición se registra una línea base de dos minutos, por lo que al final se tienen tres líneas base y tres condiciones, las cuales duran también dos minutos. La duración total del perfil es 12 minutos.

Para registrar la actividad autonómica se utilizó el equipo de retroalimentación biológica Procomp 2® y sensores para la CGP, así como el software BioGraph® Infiniti SA7900 v 5.1.2. El software y el equipo de retroalimentación se cargaron en una computadora portátil.

\section{Datos de identificación}

Se aplicó un cuestionario de cinco preguntas cerradas para conocer los siguientes datos: edad, sexo, estado civil, número de hijos y con quién vive. 


\section{Procedimiento}

Una vez autorizado el estudio por el Comité de Ética, se solicitó a algunos profesores permiso para visitar a los alumnos en cada grupo durante su hora de clase y explicarles el estudio, leerles el consentimiento informado, aplicar los inventarios de Beck y agendar una cita en horarios que también fueron aceptados por los profesores de las asignaturas afectadas. Se pidió a los estudiantes que fueran irregulares no participar en el estudio y fueron retirados del salón.

Los inventarios fueron aplicados de modo grupal por cuatro estudiantes de la misma licenciatura en Enfermería y Obstetricia, las cuales desarrollaban actividades de investigación como parte de su servicio social universitario. Antes de aplicar las evaluaciones o el perfil psicofisiológico, las alumnas recibieron la capacitación adecuada por parte del investigador principal, el cual es también responsable del programa de servicio social en el cual ellas se desempeñaban. La aplicación se efectuó siguiendo el protocolo descrito en la tabla 1.

Tabla 1.

Protocolo para reclutar participantes y aplicar inventarios.

Actividad Tiempo

Saludar al grupo, presentarse y explicar los objetivos del estudio.

05 minutos

Entregar el consentimiento informado, leerlo, aclarar dudas y obtener firma.

10 minutos

Entregar, explicar y completar entrevista sociodemográfica.

05 minutos

Explicar la manera de completar el inventario de ansiedad de Beck y entregar 05 minutos

los instrumentos para su llenado.

Llenado del inventario de ansiedad.

30 minutos

Explicar la manera de completar el inventario de depresión de Beck y entregar 05 minutos los instrumentos para su llenado.

Llenado del inventario de depresión.

30 minutos

Informar cita para evaluación psicofisiológica.

05 minutos

Tiempo total 95 minutos

El perfil psicofisiológico se elaboró en un consultorio equipado con un escritorio, un diván y dos sillas de visita. El participante acudía a la evaluación psicofisiológica según la cita asignada y era recibido por una estudiante prestadora de servicio social y se aplicaba el protocolo que se muestra en la tabla 2 (Ruvalcaba \& Domínguez, 2011).
Tabla 2.

Protocolo para aplicar el perfil psicofisiológico de estrés.

\section{ACtividAD}

TIEMPO

Recibir al paciente y pedirle que se recostara en el diván.

Explicarle el procedimiento de registro de datos y las condiciones del perfil psicofisiológico.

Limpiar el área y colocar los sensores de conductancia galvánica de la piel.

05 minutos

Iniciar el software con el perfil psicofisiológico.

05 minutos

Desconectar al paciente y agradecer su participación

Tiempo total 30 minutos

\section{Mediciones}

\section{Inventario de ansiedad de Beck}

Se considera que existe ansiedad si la suma de las respuestas (puntaje crudo) es mayor a 16 puntos (tabla 3 ).

\section{Inventario de depresión de Beck}

El nivel se indica a partir de las puntuaciones crudas obtenidas. Se considerará que hay depresión si el puntaje es mayor a 17 (tabla 3 ).

Tabla 3.

Nivel de ansiedad y depresión según puntaje crudo.

\begin{tabular}{lcc|} 
& \multicolumn{2}{c}{ Puntaje Crudo } \\
\cline { 2 - 3 } Nivel & Ansiedad & Depresión \\
\hline Mínimo & $0-5$ & $0-9$ \\
\hline Leve & $6-15$ & $10-16$ \\
\hline Moderado & $16-30$ & $17-29$ \\
Severo & $31-63$ & $30-63$
\end{tabular}

\section{Actividad autonómica}

La señal registrada fue la CGP, que se utiliza como un indicador de activación simpática, es decir, de estrés (Treister et al., 2012).

Para este estudio se consideró que la actividad autonómica es la diferencia entre los puntajes medios de conductancia galvánica de la piel obtenidos en la línea base 1 (LB1) menos la obtenida en la condición de RNR. Entre más pe- 
queña sea la diferencia, o que resulte negativa, se considera una actividad autonómica asociada al control del estrés.

\section{Datos de identificación}

Se utilizaron frecuencias para conocer la cantidad de personas que había en cada una de las variables (edad, sexo, estado civil, número de hijos, y con quien vive).

\section{Análisis estadístico}

Para las variables sociodemográficas, los niveles de ansiedad y depresión y para los niveles de actividad autonómica entre las diferentes condiciones del perfil psicofisiológico se utilizaron estadísticas descriptivas.

Para comparar los niveles de ansiedad y depresión en la población estudiada se usó una $r$ de Spearman. Para comparar las diferencias entre los niveles de ansiedad y depresión entre el sexo y el nivel escolar se usó una $X^{2}$ (chi cuadrada).

Para evaluar los cambios en la actividad autonómica entre las diferentes condiciones se usó una prueba de Kruskal-Wallis; para evaluar las diferencias entre condiciones por sexo, se utilizó una t de Student para grupos independientes. Por último se utilizó una prueba de ANOVA para comparar las variables sociodemográficas con el estado emocional (ansiedad y depresión) y con la actividad autonómica. Asimismo se usó una $r$ de Spearman para hacer correlaciones entre las variables sociodemográficas y el estado emocional (ansiedad y depresión) y los niveles de actividad autonómica.

\section{Resultados}

\section{Muestra}

En el estudio fueron incluidos sólo los estudiantes regulares de los tres primeros grados del programa educativo de licenciatura en Enfermería de una universidad de Guanajuato, México. Los alumnos de los últimos dos grados no fueron incluidos por estar fuera de la institución durante el estudio. Así, el total de alumnos en los tres primeros grados de la licenciatura fue de 189; sin embargo, cuando se aplicaron los inventarios, 25 personas no asistieron a clase por motivos personales o no eran alumnos regulares, por lo que no fueron evaluados.

Así, se aplicaron los inventarios de ansiedad y depresión de Beck a 164 personas, a las cuales se les asignó una cita para la evaluación psicofisiológica. De esta población se excluyeron las mediciones de 31 participantes, basados en tres criterios: 1) había preguntas sin contestar en el cuestionario de datos sociodemográficos; 2) los inventarios de ansiedad y/o depresión de
Beck indicaban más de una opción en alguna pregunta, o preguntas sin contestar, y 3) inasistencia a la evaluación psicofisiológica. Por tanto, al final se analizaron los datos de 133 alumnos (figura 1).
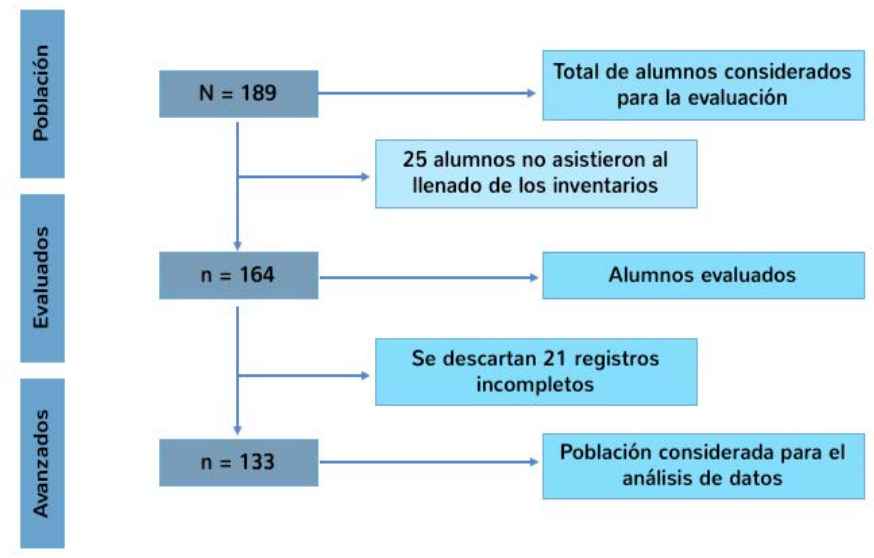

Figura 1.

Diagrama de flujo participantes.

Nota: Los alumnos incluidos en el estudio cursan los semestres primero, tercero y quinto. Los alumnos de los últimos semestres de la formación (séptimo y noveno) no fueron incluidos por estar desarrollando prácticas y el servicio profesional respectivamente

\section{Participantes}

De los 133 participantes, 108 eran mujeres (81.2\%) y 25 hombres $(18.8 \%)$, con una edad entre 18 y 23 años, media 19.3 años $(D S \pm 1.2), 126$ personas $(94.7 \%)$ eran solteros y sólo seis indicaron estar casados (4.5\%) o vivir en unión libre (un participante, $0.8 \%$ ).

\section{Tabla 4.}

Características sociodemográficas por grado escolar.

\begin{tabular}{|c|c|c|c|}
\hline \multirow{2}{*}{ VARIABLE } & \multicolumn{3}{|c|}{ Semestre } \\
\hline & Primero & SEgundo & TERCERO \\
\hline $\mathrm{n}$ & 47 & 41 & 45 \\
\hline Edad & $\begin{array}{c}18.3 \text { años } \\
( \pm 0.54)\end{array}$ & $\begin{array}{c}19.3 \text { años } \\
( \pm 0.73)\end{array}$ & $\begin{array}{c}20.4 \text { años (土 } \\
1.0)\end{array}$ \\
\hline Sexo & $\begin{array}{c}36(76.6 \%) \\
\text { mujeres }\end{array}$ & $\begin{array}{c}33(80.5 \%) \\
\text { mujeres }\end{array}$ & $\begin{array}{c}39(86.7 \%) \\
\text { mujeres }\end{array}$ \\
\hline Estado civil & $\begin{array}{c}46(97.9 \%) \\
\text { solteros }\end{array}$ & $\begin{array}{c}41(100 \%) \\
\text { solteros }\end{array}$ & $\begin{array}{c}39(86.7 \%) \\
\text { solteros }\end{array}$ \\
\hline Vive con & $\begin{array}{c}38(80.9 \%) \\
\text { Padres }\end{array}$ & $\begin{array}{c}33(80.5 \%) \\
\text { Padres }\end{array}$ & $\begin{array}{c}32(71.1 \%) \\
\text { Padres }\end{array}$ \\
\hline Núm. de hijos & 0 hijos & 0 hijos & 0.2 hijos \\
\hline
\end{tabular}

Nota: Los valores fuera del paréntesis indican número de participantes, excepto en Edad (años) y en Núm. de hijos (hijos). En el caso de la Edad el número entre paréntesis indica la desviación estándar. 
La mayoría (103 personas, $77.4 \%$ ) vive con sus papás, 22 participantes viven con otras personas $(16.6 \%)$, siete con su familia de procreación $(5.3 \%)$ y sólo una persona $(0.8 \%)$ manifestó vivir sola. La mayoría (124 participantes, $93.2 \%)$ no tiene hijos, siete personas (5.3\%) tiene uno, y solamente en dos casos, las participantes indicaron tener dos hijos.

Respecto al nivel educativo, 47 individuos (35.3\%) pertenecían al primer semestre; $41(30.8 \%)$ al tercero y $45(33.8 \%)$ al quinto (tabla 4$)$.

\section{Estado emocional}

\section{Ansiedad}

El puntaje crudo mínimo fue 12 y el máximo 58. La media fue 23.17 ( $D S \pm 7.1$ ), lo que corresponde a un nivel moderado de ansiedad; esto es, 87 personas (65\%) puntuó en este nivel; 28 personas $(21 \%)$ mostraron niveles leves y $18(13.5 \%)$ calificó en nieles severos de ansiedad (figura 2). No se encontraron relaciones significativas entre los niveles de ansiedad y depresión de los participantes $\left(r_{s}=0.077, p=0.380\right)$.

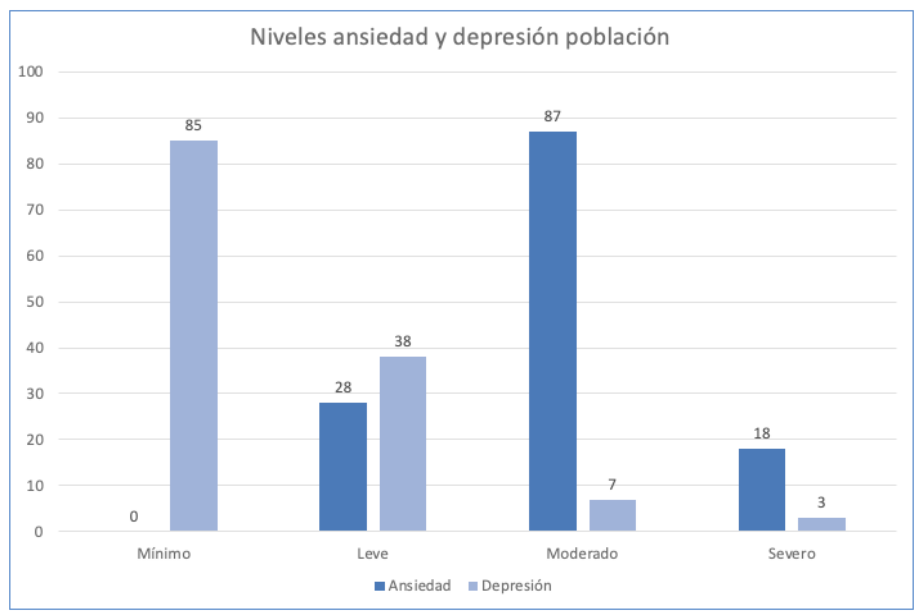

Figura 2.

Niveles de ansiedad y depresión en la población estudiada.

Tanto en hombres como en mujeres los niveles de ansiedad tuvieron índices similares: $20 \%$ de los hombres y $21.3 \%$ de las mujeres mostraron niveles leves. En el nivel moderado puntuaron $68 \%$ de los hombres y $64.8 \%$ de las mujeres. Respecto al nivel severo de ansiedad, refirieron padecerlo $12 \%$ de los hombres y $13.9 \%$ de las mujeres. No se registraron niveles mínimos de ansiedad.

No se encontraron diferencias significativas entre los niveles de ansiedad de hombres y mujeres (X2 $=0.001, \mathrm{gl}=$ $1, p=0.973)$. Analizando los datos por semestre, tampoco se encontraron diferencias significativas en los niveles de ansiedad de los participantes $\left(X^{2}=0.308, g /=2, p=0.857\right)$.

\section{Depresión}

En la figura 2 también se muestran los niveles de depresión en la población evaluada. El puntaje crudo mínimo fue 0 , el máximo 34 y la media $7.9(D S \pm 6.9)$. Así, casi todos los que respondieron el inventario puntuaron en niveles mínimos de depresión (85 personas, 63.9\%), o leves (38 estudiantes, 28.6\%). En el nivel moderado puntuaron siete participantes (5.2\%), siendo el nivel severo el que menos se presentó (tres personas, 2.3\%).

Catorce hombres (56\%) indicaron niveles mínimos de depresión, mientras que 71 mujeres $(65.7 \%)$ puntuaron en el mismo nivel. En el nivel leve se encontraron ocho hombres $(32 \%)$ y 30 mujeres $(27.8 \%)$. El $8 \%$ de los hombres (dos individuos) indicaron niveles moderados de depresión, mientras que $4.6 \%$ de las mujeres (cinco) se localizaron en este nivel. Sólo un hombre $(4 \%)$ y dos mujeres (1.9\%) indicaron niveles severos de depresión (tabla 5).

\section{Tabla 5}

Niveles de ansiedad y depresión por sexo.

\begin{tabular}{lcccc} 
& \multicolumn{2}{c}{ Ansiedad } & \multicolumn{2}{c}{ Depresión } \\
\hline $\begin{array}{l}\text { Nivel estado } \\
\text { Emocional }\end{array}$ & Hombres & Mujeres & Hombres & Mujeres \\
\hline Mínimo & 0 & 0 & 56 & 66 \\
\hline Moderado & 20 & 21 & 32 & 27 \\
\hline Leve & 68 & 65 & 8 & 5 \\
\hline Severo & 12 & 14 & 4 & 2 \\
\hline Nota: Los valores corresponden a porcentajes. &
\end{tabular}

No se encontraron diferencias significativas entre los niveles de depresión de hombres y mujeres $\left(X^{2}=\right.$ $1.029, g /=1, p=0.310$ ). Tampoco se encontraron diferencias significativas respecto a los niveles de depresión por semestre $\left(X^{2}=3.623, g l=2, p=0.163\right)$.

\section{Actividad autonómica}

Los valores de CGP que presentaron los participantes durante las diferentes condiciones que conformaron la evaluación psicofisiológica se muestran en la tabla 6 .

En dicha tabla puede verse que los valores promedio de la línea base 1 (LB1) fue de $1.92 \mu \mathrm{S}$; durante el estrés cognitivo (Stroop) se elevó a $2.88 \mu \mathrm{S}$, para descender a $2.76 \mu \mathrm{S}$ en la segunda línea base (LB2). Durante la evocación emocional (EE) los registros presentaron un ligero decremento para situarse en $2.74 \mu \mathrm{S}$. En la tercera línea base (LB3) los registros tendieron a decrecer, pues se ubicaron en $2.69 \mu \mathrm{S}$. Durante la condición de respuesta natural de relajación (RNR) prácticamente no 
hubo variación, ya que los registros mostraron un ligero decremento a $2.68 \mu \mathrm{S}$.

\section{Tabla 6}

Valores registrados por la población en el perfil psicofisiológico.

\begin{tabular}{lcccccc} 
& LB1 & StRoOP & LB2 & EE & LB3 & RNR \\
\hline Media & 1.92 & 2.88 & 2.76 & 2.74 & 2.69 & 2.68 \\
DE & 1.92 & 2.23 & 2.29 & 2.27 & 2.28 & 2.22 \\
Mínimo & 0.08 & 0.34 & 0.36 & 0.42 & 0.07 & 0.34 \\
\hline Máximo & 9.67 & 9.95 & 9.98 & 9.82 & 9.94 & 9.96
\end{tabular}

Nota: $\mathrm{DE}=$ Desviación Estándar. Los valores corresponden a Micro Siemens $(\mu S)$.

Estadísticamente sólo fueron significativos los cambios que se presentaron ente la LB1 y la condición de Stroop $(z=-9.99, p=0.00)$ y entre la condición de Stroop y la LB2 $(z=-3.862, p=0.00)$. Entre las demás condiciones los cambios no fueron significativos $(p=0.05)$. Sin embargo se observó que el nivel de activación autonómica al inicio del perfil psicofisiológico del estrés fue significativamente menor que al final de éste (RNR), lo que indica que las personas permanecen activadas después de enfrentar un estresor $(z=-7.77, p=0.00)$ (figura 3).

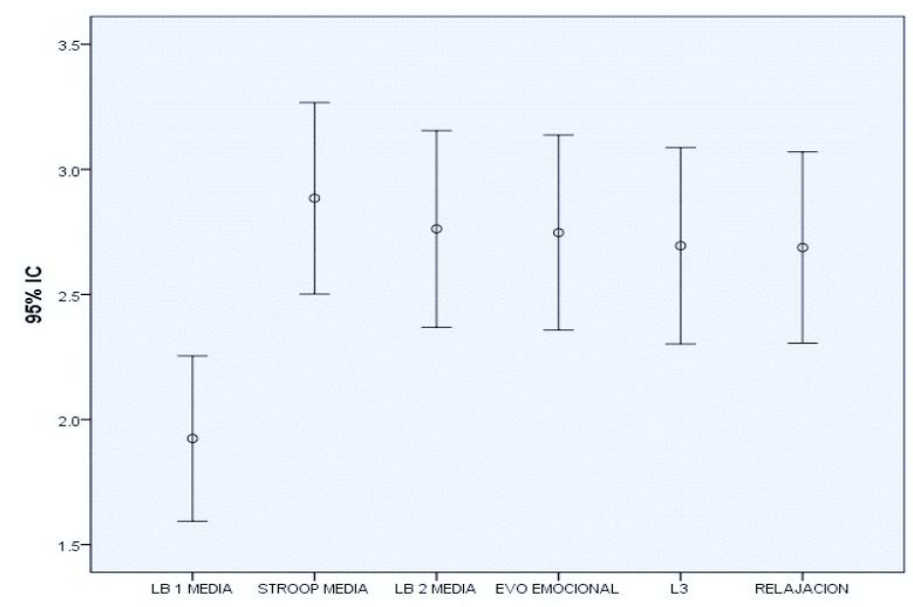

Figura 3.

Barras de error con los puntajes obtenidos en el perfil psicofisiológico.

Nota: El promedio de la actividad autonómica al inicio de la evaluación (LB1) fue significativamente menor que al final de esta (RNR), lo que indica que las personas tienden a permanecer estresadas ( $\mathrm{z}=$ -7.77, $p=.00)$. Los valores corresponden a Micro Siemens $(\mu S)$.

Cuando los registros se agrupan por sexo, el valor mínimo que presentaron las mujeres en la primera condición (LB1) fue $0.080 \mu \mathrm{S}$, el máximo $9.67 \mu \mathrm{S}$, con una media de $2 \mu \mathrm{S}(D S \pm 2.02 \mu \mathrm{S})$, mientras que en la RNR el valor mínimo fue $0.34 \mu \mathrm{S}$, el máximo $9.96 \mu \mathrm{S}$ y la media $2.79(D E \pm 2.30 \mu \mathrm{S})$. Al igual que en la población general, entre las mujeres se pudo observar que permanecen activadas simpáticamente de manera significativa entre la primera condición (LB1) y la última, es decir, en la $\operatorname{RNR}(z=-6.79, \mathrm{p}=0.00)$.

En la primera condición (LB1) el registro mínimo entre los hombres fue $0.120 \mu \mathrm{S}$, el máximo $4.92 \mu \mathrm{S}$, con una media de $1.56 \mu \mathrm{S}(D S \pm 1.37 \mu \mathrm{S})$. Por último, en la condición de RNR el valor mínimo fue $0.49 \mu \mathrm{S}$, el máximo $6.18 \mu \mathrm{S}$, la media $2.22 \mu \mathrm{S}$ y la $\mathrm{DS} \pm 1.81 \mu \mathrm{S}$, por lo que fue posible observar también que los hombres permanecen activados simpáticamente entre la primera condición (LB1) y la última RNR $(z=-3.97, p=0.00)$.

No se encontraron diferencias significativas entre los puntajes de hombres y mujeres en las distintas condiciones del perfil (tabla 7).

\section{Tabla 7}

Diferencias en las puntuaciones promedio obtenidas en el perfil por sexo.

\begin{tabular}{llll|}
\hline \multicolumn{1}{c}{ Condición } & $\begin{array}{c}\text { LEVENE } \\
\text { (SIG.) }\end{array}$ & T & SIG. BILATERAL \\
\hline Línea base 1 & 0.328 & -1.034 & 0.303 \\
\hline Stroop & 0.543 & -0.887 & 0.377 \\
\hline Línea base 2 & 0.194 & -1.015 & 0.312 \\
\hline $\begin{array}{l}\text { Evocación } \\
\text { emocional }\end{array}$ & 0.294 & -0.867 & 0.387 \\
\hline $\begin{array}{l}\text { Línea base 3 } \\
\text { Respuesta }\end{array}$ & 0.266 & -0.981 & 0.329 \\
relajación & 0.285 & -1.150 & 0.252 \\
\hline
\end{tabular}

Nota: Resultados de la prueba t para grupos independientes, comparando los promedios obtenidos en el perfil por sexo. La probabilidad, tanto en la prueba t como en la prueba de Levene para la homogeneidad de las varianzas es de 0.05 . No hay diferencias significativas en los resultados obtenidos por hombres o por mujeres.

Por otro lado, si se divide la población por grado escolar, al igual que en la población general, los participantes del primer semestre mostraron incrementos significativos entre la LB1 y la RNR $(z=-4.67, p=0.0)$. En el tercer semestre los participantes mostraron incrementos significativos en la actividad simpática entre el inicio de la intervención y al final de la misma, esto es, entre la LB1 y la RNR ( $z=-4.16, p=0.0)$. Respecto al quinto semestre, al igual que en la población general se registraron incrementos significativos entre la LB1 y la $\operatorname{RNR}(z=-4.56, p=0.0)$.

Los grupos mostraron diferencias significativas en las condiciones de LB1 $(F=3.107 ; g l=2 ; 130 ; p=$ 
$0.048), \mathrm{EE}(F=3.343 ; g l=2 ; 130 ; p=0.038), \mathrm{LB} 3(F=$ $3.433 ; g l=2 ; 130 ; p=0.035)$, y en la $\operatorname{RNR}(F=3.723 ; g l$ $=2 ; 130 ; p=0.027)$.

Estas diferencias se dieron entre los grupos tercero y quinto, con una $p=0.010$, para la LB $1 ; p=0.022$, para el EE; $p=0.021$, para la LB3 y $p=0.018$ para la RNR. El grupo de primero no mostró diferencias significativas respecto a los otros dos grupos $(p=0.05)$.

\section{Análisis multivariados, regresiones, correlaciones}

No se encontraron relaciones significativas entre variables socioeconómicas (edad, semestre y sexo) y la presencia de ansiedad, depresión o el nivel de activación autonómica. Tampoco entre niveles de actividad autonómica y la presencia de ansiedad o depresión $(p=0.05)$ (tabla 8).

Tabla 8

Anova: factores sociodemográficos, ansiedad y depresión, y actividad autonómica

\begin{tabular}{|c|c|c|c|c|c|c|c|c|c|}
\hline & & Sexo & EDAD & $\begin{array}{l}\text { SEMESTRE } \\
\text { QUE CURSA }\end{array}$ & $\begin{array}{l}\text { ANSIEDAD } \\
\text { NIVEL }\end{array}$ & $\begin{array}{c}\text { DePRESIÓN } \\
\text { NIVEL }\end{array}$ & LB1 MEDIA & $\begin{array}{l}\text { RNR } \\
\text { MEDIA }\end{array}$ & $\begin{array}{c}\text { DIF } \\
\text { LB1-RNR }\end{array}$ \\
\hline \multirow[t]{2}{*}{ Sexo } & $\begin{array}{l}\text { Coeficiente de } \\
\text { correlación }\end{array}$ & 1.000 & 0.151 & 0.107 & 0.003 & -0.088 & 0.089 & 0.097 & .046 \\
\hline & Sig. (bilateral) & - & 0.082 & 0.221 & 0.973 & 0.312 & 0.307 & 0.265 & .598 \\
\hline \multirow{2}{*}{ Edad } & $\begin{array}{l}\text { Coeficiente de } \\
\text { correlación }\end{array}$ & 0.151 & 1.000 & $0.846^{* *}$ & -0.095 & 0.064 & 0.106 & 0.010 & -.054 \\
\hline & Sig. (bilateral) & 0.082 & - & 0.000 & 0.279 & 0.463 & 0.227 & 0.910 & .539 \\
\hline \multirow{2}{*}{$\begin{array}{l}\text { Semestre } \\
\text { que cursa }\end{array}$} & $\begin{array}{l}\text { Coeficiente de } \\
\text { correlación }\end{array}$ & 0.107 & $0.846^{* *}$ & 1.000 & -0.048 & 0.081 & 0.168 & 0.069 & -.001 \\
\hline & Sig. (bilateral) & 0.221 & 0.000 & - & 0.581 & 0.353 & 0.053 & 0.433 & .987 \\
\hline \multirow{2}{*}{$\begin{array}{l}\text { Ansiedad } \\
\text { nivel }\end{array}$} & $\begin{array}{l}\text { Coeficiente de } \\
\text { correlación }\end{array}$ & 0.003 & -0.095 & -0.048 & 1.000 & 0.077 & -0.063 & 0.016 & .077 \\
\hline & Sig. (bilateral) & 0.973 & 0.279 & 0.581 & - & 0.380 & 0.470 & 0.854 & .380 \\
\hline \multirow{2}{*}{$\begin{array}{l}\text { Depresión } \\
\text { nivel }\end{array}$} & $\begin{array}{l}\text { Coeficiente de } \\
\text { correlación }\end{array}$ & -0.088 & 0.064 & 0.081 & 0.077 & 1.000 & -0.021 & -0.069 & -.012 \\
\hline & Sig. (bilateral) & 0.312 & 0.463 & 0.353 & 0.380 & - & 0.806 & 0.429 & .890 \\
\hline
\end{tabular}

Nota: ANOVA utilizando coeficiente de correlación Rho de Spearman, $N=133$, LB1: condición inicial del perfil psicofisiológico denominada línea base inicial, RNR: condición final del perfil psicofisiológico conocida como respuesta natural de relajación.

**. La correlación es significativa al nivel 0.01 (bilateral).

Se identificaron correlaciones positivas de gran magnitud entre los puntajes iniciales del perfil (LB1) y los obtenidos en la RNR $\left(r_{s}=.844, p<0.05\right)$, de manera que entre más elevados sean los puntajes al inicio tenderán a permanecer elevados al final del mismo.
También se encontraron correlaciones positivas, aunque de poca intensidad, entre la actividad autonómica en la RNR y la cantidad de cambios entre el inicio del perfil y su finalización $\left(r_{s}=0.210, p<0.05\right)$. Entre más se relaja la persona, más cambios se obtienen entre el inicio de perfil y la finalización del mismo.

En resumen, las personas estresadas al inicio tenderán a permanecer estresadas y se relajarán menos.

\section{Discusión}

En el presente estudio participaron alumnos regulares de primero, tercero y quinto semestres de la carrera de Enfermería de una universidad de Guanajuato. En estos grupos no se encontraron niveles elevados de ansiedad o depresión, pero sí niveles elevados de actividad autonó- 
tes sienten y piensan (niveles de actividad emocional) y lo que el cuerpo hace (niveles de actividad autonómica).

Otras investigaciones (Kauffman, 2016) han revelado que practicar yoga puede decrecer la ansiedad asociada al tartamudeo, sobre todo porque se decrece la actividad autonómica. Los mismos resultados se han obtenido otras investigaciones (Servinc et al., (2018), lo que no es consistente con nuestros hallazgos.

En este sentido Sánchez, Figueroa, Cacho y Robles (2016) indican que hay correlación entre los síntomas autonómicos y la presencia de ansiedad y depresión en pacientes con cáncer de mama. Infortunadamente los síntomas que ellos miden son autonómicos en general, con pacientes que padecen cáncer, y sus resultados no están determinados a partir de la aplicación de equipos de retroalimentación biológica con personas sanas.

Por otro lado, es posible que la sostenida activación fisiológica que se registró en las personas estudiadas se deba a la novedad del estímulo; algunos investigadores (Riedl y Fischer, 2018) mencionan que el tiempo es un factor que debe tenerse en cuenta si no se quiere que los participantes permanezcan activados a lo largo del perfil psicofisiológico, pero esto es algo que debe revisarse en otras investigaciones.

También es posible que la falta de relación entre la actividad autonómica y los niveles de ansiedad y/o depresión se deban a que no se consideran factores de naturaleza no psicológica, como puede ser la alimentación, las bebidas, la actividad antes de cada medición, la interacción con otras personas e incluso la respiración, tal como lo proponen algunos autores (Sperry, Kwapil, Eddington y Silva, 2018).

Además, aunque algunos autores indican que la activación simpática crónica se correlaciona de modo directo con el desarrollo de ansiedad y depresión (Streeter et al., 2012), nuestro estudio demuestra que no hay relación entre la actividad autonómica y los niveles de estos trastornos emocionales en las personas incluidas, por lo que esto es algo que también debe investigarse más.

Por otro lado, es probable que los alumnos de los últimos semestres hayan mostrado mayores niveles de actividad autonómica (es decir, mayores niveles de estrés) porque se ha encontrado que estos alumnos combinan normalmente sus actividades escolares con otras obligaciones, como trabajar o desempeñarse como padres (Kornguth, Frisch, Shovein y Williams, 1984); en nuestra población, en el grupo de quinto semestre había más alumnos que eran padres o que vivían con su familia de procreación, o que trabajaban. Sin embargo, estos índices no fueron significativos, aunque esto es algo que debe considerarse para próximas investigaciones.
En cuanto al estado emocional, nuestros resultados indican que tanto hombres como mujeres tienden a presentar más ansiedad que depresión. Sin embargo, los niveles con que se registraron estas alteraciones no fueron muy elevados; aunque la ansiedad estuvo presente en la mayoría de las personas $(67 \%$ de quienes fueron incluidos tuvieron nivel moderado, mientras que $21 \%$ tuvo nivel leve), los niveles de depresión fueron mínimos en su mayoría, pues $70 \%$ de los participantes así lo refirió, y el restante 30\% indicó que la depresión estuvo presente sólo en un nivel leve; por tanto, estos índices no fueron suficientes como para indicar que los estados de ansiedad o depresión estuvieron en nuestra población de manera significativa.

No obstante, estudios nacionales e internacionales han documentado que la ansiedad y la depresión son trastornos comunes en los estudiantes del nivel superior. Por ejemplo, reportes administrativos acerca de consultas para el apoyo emocional al estudiante universitario en la Universidad Industrial de Santander (UIS) indican que 9\% de los casos atendidos corresponden al trastorno de ansiedad, y en la Universidad Pontificia Bolivariana de Bucaramanga (UPB) del total de la población atendida, $17 \%$ fue por presentar rasgos ansiosos y $12 \%$ por depresión (Agudelo et al., 2008). Sin embargo, en otros estudios se ha documentado que la prevalencia de depresión entre estudiantes de diferentes carreras en Colombia es de $49.8 \%$ y de $58.1 \%$ para la ansiedad (Amézquita, González y Zuluaga, 2003). Estos autores también documentaron que los factores sociodemográficos son condicionantes para padecer alteraciones emocionales. Por ejemplo, el compartir el departamento con compañeros de escuela era factor de riesgo para depresión y ansiedad, y que el estar en primer año y provenir de un estrato económico bajo eran factores de riesgo para padecer ansiedad.

En este sentido es probable que las personas padezcan alteraciones emocionales cuando cursan los primeros semestres de una carrera en el área de la salud, como lo demuestran Aragón, Chávez y Flores (2015) al mencionar que entre 12 y $19 \%$ de los estudiantes de Cirujano Dentista de una universidad de México presentaban ansiedad, y que la población de los primeros semestres presentaban mayores niveles si se le comparaba con estudiantes de niveles más altos. Esto es consistente con nuestros resultados.

Respecto a la ansiedad y depresión como trastornos que se presentan comórbidos debido a factores neurobiológicos comunes, encontramos que no hubo relación entre la presencia de ansiedad o depresión; sin embargo, hay importante evidencia científica que indica que las condiciones patológicas de ansiedad y depresión comparten similares procesos etiológicos y de perma- 
nencia, así como similares factores de riesgo familiares, genéticos y ambientales (Newby, McKinnon, Kuyken, Gilbody y Dalgleish, 2015). También se ha documentado que ambos padecimientos comparten los mismos componentes afectivos negativos y factores de estrés, así como similares factores de mantenimiento emocionales, cognitivos y conductuales. Es más, otras investigaciones han encontrado que existen relaciones significativas de hasta $90 \%$ entre estos trastornos, de manera que los pacientes diagnosticados con ansiedad también presentan depresión (Muñoz, et al., 2005), por lo que sugerimos desarrollar más investigación al respecto.

Así, y a pesar de lo encontrado en nuestro estudio, no podemos negar que la ansiedad y la depresión son padecimientos muy prevalentes que ocasionan deterioro psicológico, físico y social, cuya incidencia va en aumento, sobre todo entre la población estudiantil universitaria, es decir, entre los que serán los líderes del futuro, los que tomarán decisiones y conformarán el progreso de nuestra sociedad. Es urgente implementar acciones que permitan ofrecer opciones de tratamiento y prevención eficaces y de bajo costo que contribuyan con efectividad a detener su incidencia y prevalencia; pero se necesita más investigación que incluya más carreras del ámbito de la salud y que considere el tiempo como una variable indispensable.

Es necesario indicar que, dentro de la Psicología, las intervenciones cognitivo conductuales han demostrado ser eficaces para el tratamiento de estos problemas (Regehr et al., 2013), sobre todo mediante el uso de la retroalimentación biológica (Chittaro y Sioni, 2014; Cutshall, et al., 2011), tal como lo demostramos nosotros. Sin embargo, en nuestro país hay pocos estudios que evalúen los efectos de la aplicación de estas intervenciones entre estudiantes universitarios. Más aún, hay una creciente necesidad de información acerca de las condiciones de salud física y mental de los adolescentes y de los universitarios en particular, de manera que puedan establecerse programas de atención y prevención de alteraciones que pudieran derivar en trastornos crónicos (Denny, et al., 2014), como es el caso de la ansiedad y la depresión.

\section{Conclusiones}

En nuestro estudio no se demostró que hubiera congruencia entre el estado emocional y la actividad autonómica asociada a dicho estado, esto es, las personas no sentían padecer estrés, ansiedad o depresión, aunque el funcionamiento fisiológico indicaba que el estrés permanecía durante el tiempo que duraba el perfil psicofisiológico, estando presente aun en la condición en que se pedía a las personas se relajaran como sabían hacerlo.

Estos resultados deben ser considerados al establecer protocolos de atención para estudiantes universitarios que padecen estrés, ansiedad o depresión, pues incluir a estudiantes en intervenciones que no perciben como benéficas ocasiona que el índice de deserción clínica se incremente de manera drástica. Debe considerarse que la intervención psicofisiológica para modificar la actividad autonómica se basa en los ejercicios que los pacientes hacen en casa para lograr la generalización de la respuesta, pero si no sienten necesidad de semejantes ejercicios no los harán, priorizando otras tareas sobre dicha práctica.

Por tanto, debe asegurarse que los involucrados en una intervención sientan que padecer alteraciones emocionales son factores que pudieran afectar tanto su actividad autonómica como los resultados de su desempeño académico. Sin embargo, debe recordarse que en el estudio se incluyó a todo tipo de personas; el único requisito era que fueran alumnos regulares.

Así pues, se recomienda hacer más estudios que incluyan población clínica, es decir, población que padezca ansiedad o depresión y que tengan calificaciones que los ubiquen como estudiantes de riesgo. Las instrucciones en este estudio son fácilmente replicables y pueden desarrollarse otras investigaciones que consideren a las población sugerida, o incluso a poblaciones similares a la estudiada para de este modo conformar un cuerpo teórico que genere nuevas directrices en el comportamiento emocional o en el autonómico, pero sobre todo en el estrés académico de nuestros jóvenes universitarios.

Asimismo, es necesario controlar mejor las variables alrededor de las mediciones autonómicas y comparar entre inventarios que permitan conocer el estado emocional de las personas; esto ayudaría a obtener mejores resultados. En este sentido, es necesario hacer más estudios que permitan conocer de modo más preciso los niveles de ansiedad y depresión entre la población estudiada y cómo se relacionan con el estrés académico y con la intervención psicofisiológica.

Por último, decir que el estrés es necesario para el funcionamiento diario de las personas. De manera particular, adecuados niveles de estrés pueden ser benéficos para los universitarios, pues les ayudan a lograr sus objetivod académicos y personales (Consolo et al., 2008), pero no lo es mantenerlo de manera patológica, pues puede derivar en estados crónicos de ansiedad o depresión. 


\section{Referencias}

Abravanel, B. T., \& Sinha, R. (2015). Emotion dysregulation mediates the relationship between lifetime cumulative adversity and depressive symptomatology. Journal of Psychiatric Research, 61, 89-96. doi: 10.1016/j. jpsychires.2014.11.012.

Agudelo, V. D. M., Casadiegos, G. C. P., \& Sánchez, O. D. L. (2008). Características de ansiedad y depresión en estudiantes universitarios. International Journal of Psychological Research, 1(1), 34-39. Recuperado de https://www.redalyc. org/articulo.oa?id $=299023503006$

Amézquita, M. M. E., González, P. R. E., \& Zuluaga, M. D. (2003). Prevalencia de la depresión, ansiedad y comportamiento suicida en la población estudiantil de pregrado en la Universidad de Caldas, año 2000. Revista Colombiana de Psiquiatría, 32(4), 341-356. Recuperado de http://www.

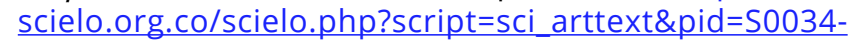
74502003000400003\&lng=en\&nrm=iso\&tlng=es

Aragón, B. L. E., Chávez, B. M., \& Flores T. M. A. (2015). Evaluación de la ansiedad en estudiantes de la carrera de Cirujano Dentista. Revista Digital Internacional de Psicología y Ciencia Social, 1(1), 33-42. Recuperado de http://cuved.unam.mx/ revistas/index.php/rdpcs/article/view/27/32

Beiter, R., Nash, R., McCrady, M., Rhoades, D., Linscomb, M., Clarahan, M., \& Sammut, S. (2015) The prevalence and correlates of depression, anxiety, and stress in a sample of college students. Journal of Affective Disorders, 173, 9096. doi: 10.1016/j.jad.2014.10.054.

Beltrán, M. D. C., Freyre, M. Á., \& Hernández-Guzmán, L. (2012). El Inventario de Depresión de Beck: Su validez en población adolescente. Terapia psicológica, 30(1), 5-13

Chan, M. F., Creedy, D. K., Chua, T. L., \& Lim C. C. (2011). Exploring the psychological health related profile of nursing students in Singapore: A cluster analysis. Journal of Clinical Nursing, 20(23-24), 3553-3560. doi:10.1111/j.13652702.2011.03807.x.

Chittaro, L., \& Sioni, R. (2014). Affective computing vs. affective placebo: Study of a biofeedback-controlled game for relaxation training. International Journal of Human-Computer Studies, 72(8-9), 663-673. doi: 10.1016/j.jijcs.2014.01.007.

Consolo, K., Fusner, S., \& Staib, S. (2008). Effects of diaphragmatic breathing on stress levels of nursing students. Teaching and Learning in Nursing, 3(2), 67-71. doi: 10.1016/j. teln.2007.10.003.

Cutshall, S. M., Wentworth, L.J., Wahner-Roedler, D. L, Vincent, A., Schmidt, J. E., Loehrer, L. L., y Bauer, B. A. (2011). Evaluation of a biofeedback-assisted meditation program as a stress management tool for hospital nurses: A pilot study. Explore, 7(2), 110-112. doi: 10.1016/j.explore.2010.12.004.

Denny, S., Silva de, M., Fleming, T., Clark, T., Merry, S., Ameratunga, S., \& Fortune, S. A. (2014). The prevalence of chronic health conditions impacting on daily functioning and the association with emotional well-being among a national sample of high school students. Journal of Adolescent Health, 54(4), 410-415. doi: 10.1016/j. jadohealth.2013.09.010.

Dieleman, G. C., Huizink, A. C., Tulen, J. H. M., Utens, E. M. W. J.,
Creemers, H. E., Van der Ende, J., \& Verhulst, F. C. (2015). Alterations in HPA-axis and autonomic nervous system functioning in childhood anxiety disorders point to a chronic stress hypothesis. Psychoneuroendocrinology, 51, 135-150. doi: 10.1016/j.psyneuen.2014.09.002.

Galindo, V. O., Rojas, C. E., Meneses, G. A., Aguilar, P. J. L., Álvarez, A. M. A., \& Alvarado, A. S. (2015). Propiedades psicométricas del Inventario de Ansiedad de Beck (BAl) en pacientes con cáncer. Psicooncología, 12(1), 51-58. doi: 10.5209/rev_PSIC.2015.v12.n1.48903.

Grobecker, A. P. (2016). A sense of belonging and perceived stress among baccalaureate nursing students in clinical placements. Nurse Education Today, 36, 178-183. doi: 10.1016/j.nedt.2015.09.015.

Ibrahim, A. K., Kelly, S. J., Adams, C. E., \& Glazebrook, C. (2013). A systematic review of studies of depression prevalence in university students. Journal of Psychiatric Research, 47(3): 391-400. doi: /10.1016/j.jpsychires.2012.11.015.

Jiménez, C., Martínez, N.-O. P., \& Vacas, D. C. (2010). Stress and health in novice and experienced nursing students. Journal of Advanced Nursing, 66(2), 442-455. doi: 10.1111/j.13652648.2009.05183.x.

Jurado, S., Villegas, M. E., Méndez, L., Rodríguez, F., Loperena, U. V., \& Varela, R. (1998). La estandarización del inventario de depresión de Beck para los residentes de la Ciudad de México. Salud Mental, 21(3), 26-31. Recuperado de http:// www.revistasaludmental.mx/index.php/salud mental// article/view/706

Kauffman, H. (2016). Yoga: Potential benefits for persons who stutter. Perceptual and motor skills, 122(1), 193-199. doi: 10.1177/0031512516628987.

Kemp, A. H., \& Felmingham, K. L. (2008). The psychology and neuroscience of depression and anxiety: Towards an integrative model of emotions disorder. Psychology \& Neuroscience, 1(2), 177-181. doi: 10.3922/j. psns.2008.2.010.

Kornguth, M., Frisch, N., Shovein, J., \& Williams, R. (1984). Noncognitive factors that put students at academic risk in nursing programs. Nurse Educator, 19(5), 24-27. doi: 10.1097/00006223-199409000-00017.

Medina-Mora, M. E., Borges, G., Benjet, C., Lara, M. C., Rojas, E., Fleiz, C, y Aguilar-Gaxiola, S. (2009). Estudio de los trastornos mentales en México: Resultados de la encuesta mundial de salud. En: J. J. Rodríguez, R. Khon \& S. AguilarGaxiola (eds.). Epidemiología de los trastornos mentales en América Latina y el Caribe (pp. 79-89). Washington, D.C., USA: Organización Panamericana de la Salud. Recuperado de http://iris.paho.org/xmlui/bitstream/ handle/123456789/740/9789275316320.pdf?sequen

Muñoz, R. A., McBride, M. E., Brnabic, A. J. M., López, C. J., Hetem, L. A. B., Secin, R., \& Dueñas, H. J. (2005). Major depressive disorder in Latin America: The relationship between depression severity, painful somatic symptoms, and quality of life. Journal of Affective Disorders, 86(1), 93-98. doi: 10.1016/j.jad.2004.12.012.

Newby, J. M., McKinnon, A., Kuyken, W., Gilbody, S., \& Dalgleish, T. (2015). Systematic review and meta-analysis of transdiagnostic psychological treatments for anxiety and depressive disorders in adulthood. Clinical Psychology 
Review, 40, 91-110. doi: 10.1016/j.cpr.2015.06.002.

Organización Panamericana de la Salud (OPS) (2017). Depresión y otros trastornos mentales comunes. Estimaciones sanitarias mundiales. Washington, D.C., USA: Autor. Recuperado de http://iris.paho.org/xmlui/bitstream/ handle/123456789/34006/PAHONMH17005-spa. pdf? sequence $=1$ \&isAllowed $=y$

Regehr, C., Glancy, D., \& Pitts, A. (2013). Interventions to reduce stress in university students: A review and meta-analysis. Journal of Affective Disorders, 148(1): 1-11. doi: 10.1016/j. jad.2012.11.026.

Riedl, R., \& Fischer, T. (2018). System response time as a stressor in a digital world: Literature review and theoretical model. En: F-H. Nah \& B. S. Xiao (eds.). $\mathrm{HCl}$ in business, government, and organizations (pp. 175-186). Cham, Suiza: Springer. doi: 10.1007/978-3-319-91716-0 14.

Robles, R., Varela, R., Jurado, S., \& Páez, F. (2001). Versión mexicana del inventario de ansiedad de Beck: Propiedades psicométricas. Revista Mexicana de Psicología, 18(2), 211-218.

Ruvalcaba, P. G., \& Domínguez, T. B. (2008). Uso de la retroalimentación biológica en el dolor crónico. Gaceta Latinoamericana del Dolor, 5(4): 4-9.

Ruvalcaba, P. G., \& Domínguez, T. B. (2011). Efectos psicológicos y físicos de la modulación autonómica en el dolor miofascial: Un estudio aleatorizado. Revista Mexicana de Análisis de la Conducta, 37(2), 99-115. doi: 10.5514/rmac. v37.i2.26142.

Ruvalcaba, P. G., \& Galván, G. A. (2017). Manual de capacitación en el uso de la retroalimentación biológica para el desarrollo de investigación clínica (pp. 107-109). Cartagena de Indias, Colombia: Sello Editorial Tecnológico Comfenalco.

Sánchez, H. M. S., Figueroa, L. C. G., Cacho, D. B., \& Robles, G. R. (2016). Relación entre síntomas autonómicos con niveles de ansiedad y depresión en mujeres con cáncer de mama. EN-CLAVES del Pensamiento, X(19), 145-162.

Sapolsky, R. M. (2003). El control del estrés. Investigación y Ciencia, 326, 60-62. Recuperado de http://www. investigacionyciencia.es/revistas/investigacion-y-ciencia/ numero/326/el-control-del-estrs-3914

Sevinc, G., Hölzel, B. K., Hashmi, J., Greenberg, J., McCallister, A., Treadway, y Lazar, S. W. (2018). Common and dissociable neural activity after mindfulness-based stress reduction and relaxation response programs. Psychosomatic Medicine, 80(5), 439-451. doi: 10.1097/ PSY.0000000000000590.
Song, Y., \& Lindquist, R. (2014). Effects of mindfulness-based stress reduction on depression, anxiety, stress and mindfulness in Korean nursing students. Nurse Education Today, 35(1), 86-90. doi: 10.1016/j.nedt.2014.06.010.

Sperry, H. S., Kwapil, R. T., Eddington, M. K., \& Silva, J. P. (2018). Psychopathology, everyday behaviors, and autonomic activity in daily life: An ambulatory impedance cardiography study of depression, anxiety, and hipomanic traits. International Journal of Psychophysiology, 129, 67-75. doi: https://doi.org/10.1016/j.ijpsycho.2018.04.008.

Streeter, C. C., Gerbarg, P. L., Saper, R. B., Ciraulo, D. A., \& Beown, R. P. (2012). Effects of yoga on the autonomic nervous system, gamma-aminobutyric-acid, and allostasis in epilepsy, depression, and post-traumatic stress disorder. Medical Hypotheses, 78(5), 571-579. doi: 10.1016/j. mehy.2012.01.021.

Treister, R., Kliger, M., Zuckerman, G., Aryeh, G. I., \& Eisenberg, E. (2012). Differentiating between heat pain intensities: The combined effect of multiple autonomic parameters. Pain, 153(9), 1807-1814. doi: 10.1016/j.pain.2012.04.008.

Van Reedt Dortland, A. K. B., Vreeburg, S. A., Giltay, E. J., Licht, C. M. M., Vogelzangs, N., y Zitman, F. G. (2013). The impact of stress systems and lifestyle on dyslipidemia and obesity in anxiety and depression. Psychoneuroendocrinology, 38(2), 209-218. doi: 10.1016/j.psyneuen.2012.05.017.

Watson, R., Yanhua, C., Ip, M. Y. K., Smith, G. D., Wong, T. K. S., \& Deary, I. J. (2013). The structure of stress: Confirmatory factor analysis of a Chinese version of the Stressors in Nursing Students Scale (SINS). Nurse Education Today, 33(2), 160-165. doi: 10.1016/j.nedt.2012.02.013.

Yusoff, M. S. B., Abdul, R. A. F. A., Baba, A. A., Ismail, S. B., Mat, P. M. N., \& Esa, A. R. (2013). Prevalence and associated factors of stress, anxiety, and depression among prospective medical students. Asian Journal of Psychiatry, 6(2): 128-133. doi: 10.1016/j.ajp.2012.09.012. 


\section{Meta-Análisis del Artículo}




\section{Dimensión Cuantitativa}

\section{Perfil de Evaluación entre pares}
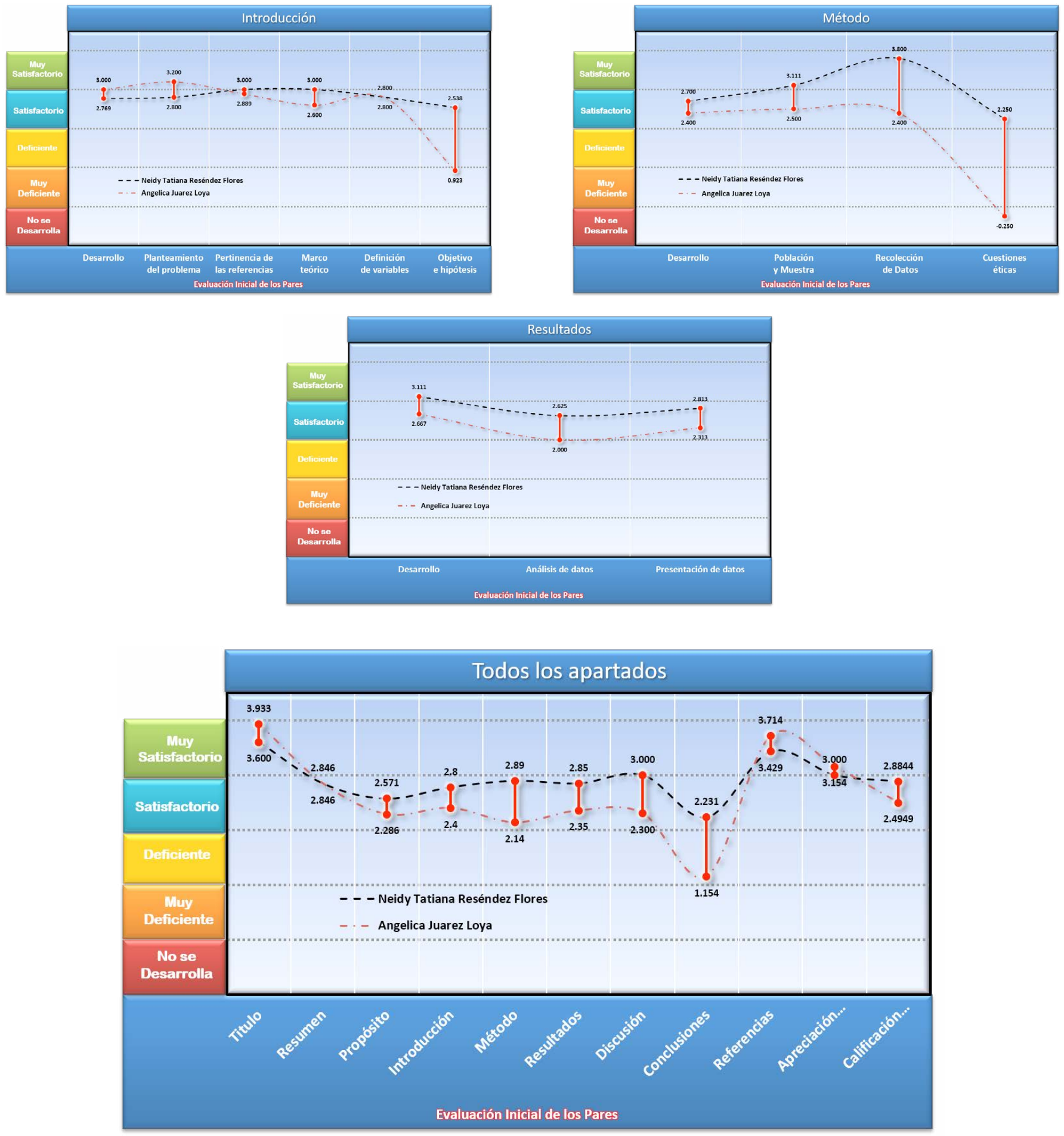


\section{Índice de Concordancia}

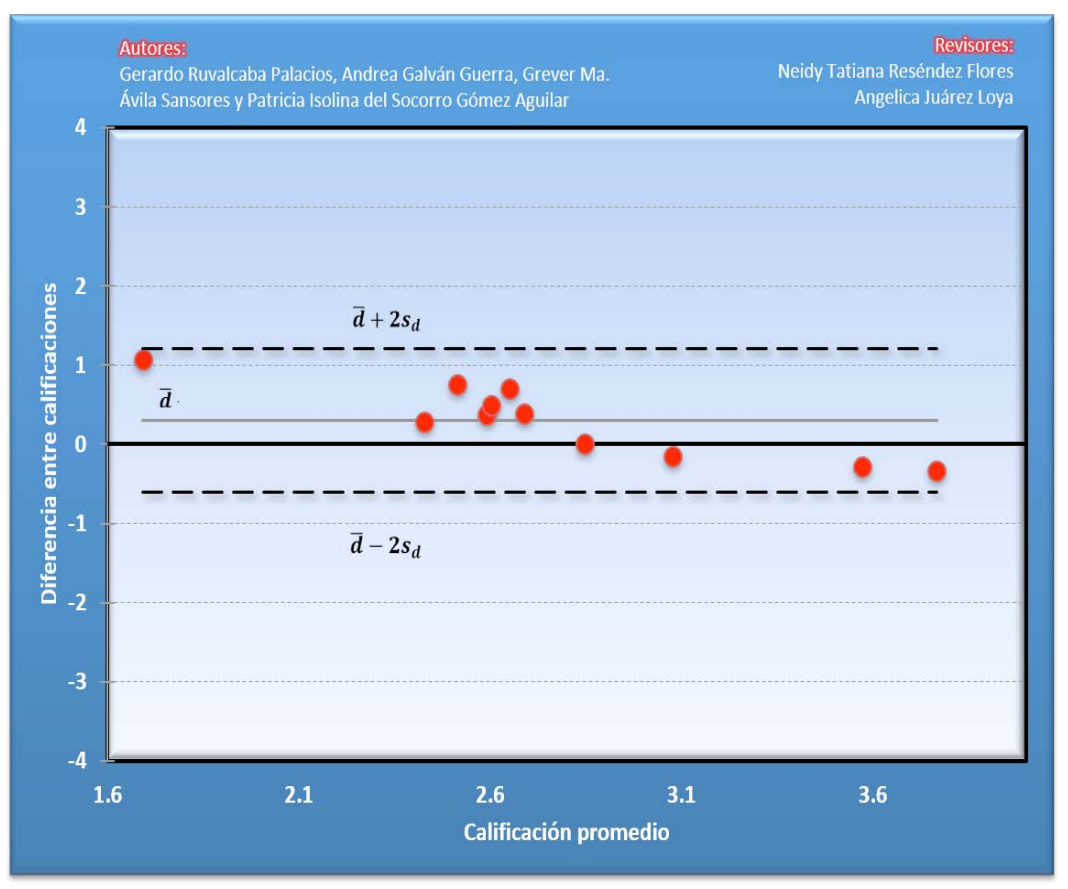

Índice de Acuerdo

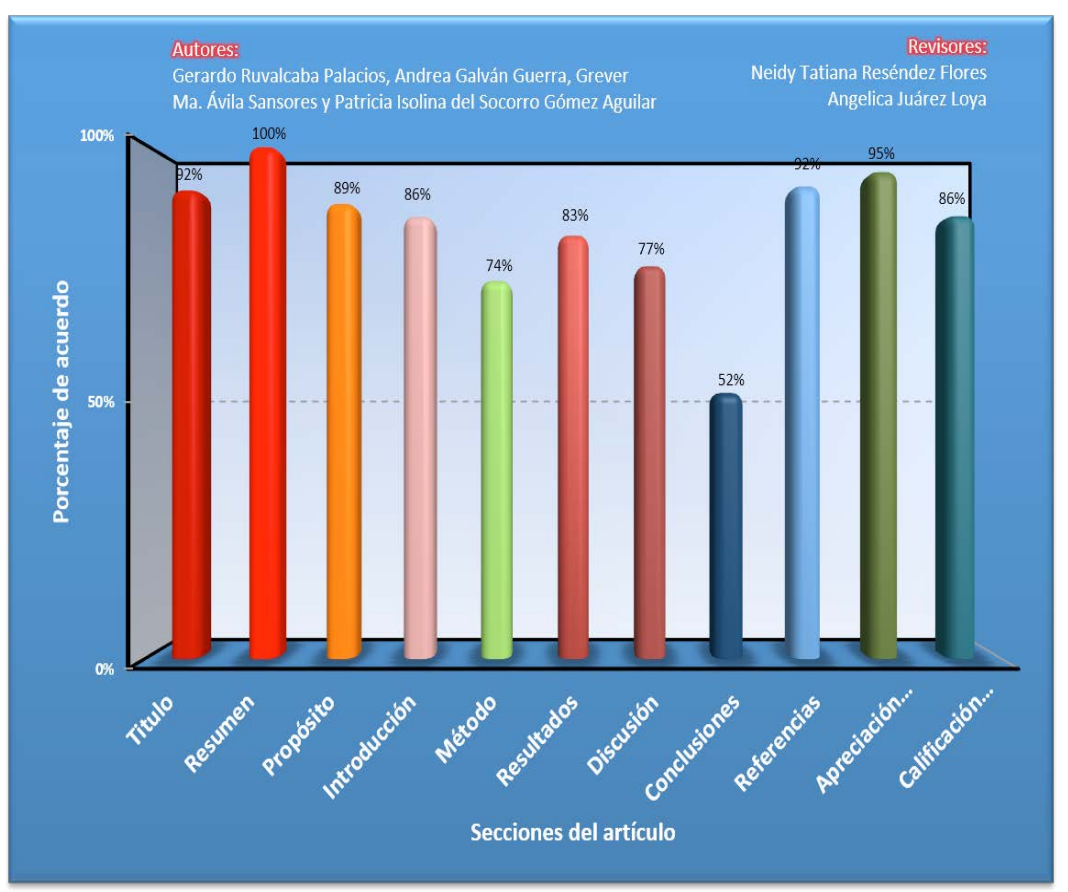




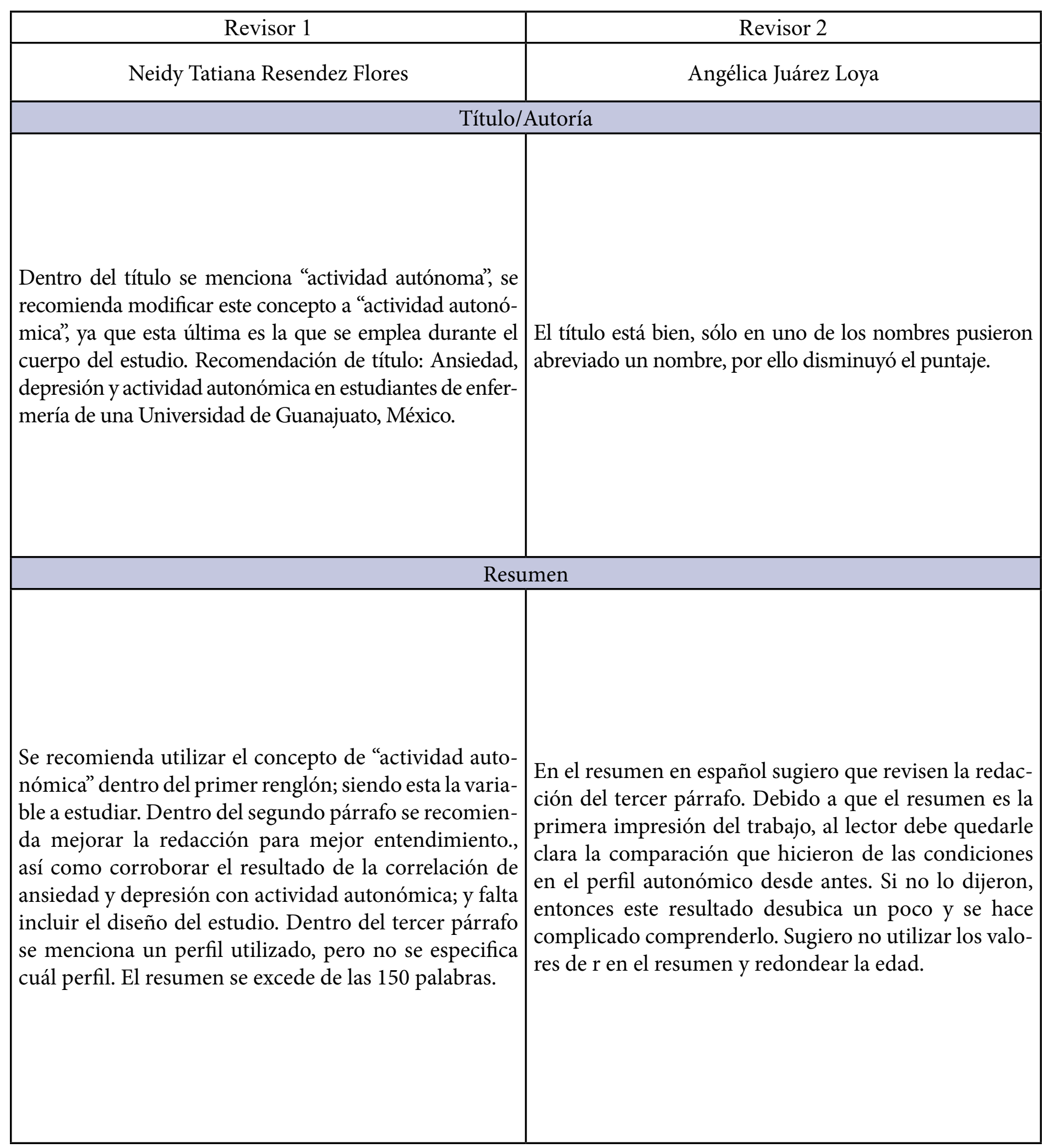




\section{Revisor 1}

Revisor 2

\section{Próposito del Estudio}

Se recomienda definir bien el objetivo del estudio, así como los objetivos específicos dentro del mismo. De acuerdo al objetivo señalado, faltan resultados de la relación entre ansiedad, depresión y actividad autonómica. Falta señalar correctamente los objetivos específicos, para que estos puedan estar ligados a los resultados expuestos; la discusión debe tener congruencia con los objetivos señalados así como con los resultados obtenidos.
La principal dificultad que observo en el objetivo es que solo dice que se haría la evaluación de las asociaciones entre variables, sin embargo en el resto del artículo (resultados y conclusiones), se presentan muchos otros análisis que no coinciden con el objetivo planteado. Sugiero que si van a reportar los análisis descritos, entonces lo enuncien en objetivos específicos. Tampoco aparece pregunta de investigación (que permita identificar las hipótesis de forma más clara) y a la hora de reportar los resultados, no muestran el resultado principal al inicio (que tendría que ser el de la correlación entre variables), tampoco aparece en la discusión el resultado principal al inicio, para darle la importancia que merece a esta pregunta de investigación que se hicieron. Sugiero que los autores evalúen también qué análisis merece la pena reportar en su artículo y cuales pueden omitirse pues ocupan gran parte de la explicación sin ser los objetivos primordiales, por ejemplo, las comparaciones por sexo quizá no son necesarias porque tienen grupos no equiparables para la comparación (por la cantidad de hombres que participaron en contraste con las mujeres), también en esta comparación es difícil que se observen diferencias por los niveles bajos de depresión que la población reportó, generalmente las diferencias solo se muestran cuando sí hay personas con depresión (igualmente con ansiedad).

\section{Introducción}

Se recomienda mencionar concretamente el objetivo general del estudio, así como los objetivos específicos del mismo, y así mismo, los resultados deben estar de acuerdo a estos objetivos. Especificar si se estudió la relación entre ansiedad, depresión y actividad autonómica, ya que no se mencionan los resultados de esta relación en los resultados, sólo los resultados de la relación entre ansiedad y depresión.
En el marco teórico, sugiero que revisen el orden de las ideas de las más generales a las ideas particulares y también que consideren incluir solo la información que deja ver al lector la importancia del estudio, incluso resaltando más una riqueza importante que tienen en sus mediciones: hicieron medición fisiológica (que no todos los estudios tienen la oportunidad de llevar a cabo). Aunque las referencias me parecen apropiadas y actuales, es necesario tratar de llevar el relato de los antecedentes con un poquito más de ilación. No tienen descritas las hipótesis. 


\begin{tabular}{|l|}
\hline \multicolumn{1}{|c|}{ Revisor 1 } \\
\hline
\end{tabular}

Sugiero que describan las propiedades psicométricas de las escalas que utilizaron, que no repitan materiales y mediciones y que amplíen la información de aspectos éticos atendiendo a los preceptos de la Declaración de Helsinky y el Código de Nurenberg. También me parece que describen con extremo detalle aspectos del procedimiento de la investigación (como lo del permiso a los profesores y afectación de sus clases) que no aportan mucho a la información que necesitamos saber como lectores, pero les falta la parte de protección de la información de los estudiantes, por poner un ejemplo. Vuelvo al punto de los análisis estadísticos, describieron muchos análisis que no están justificados en el planteamiento de su investigación y en el objetivo que describen, sería importante que todo coincidiera desde el planteamiento hasta los resultados. Respecto a la descripción de los grupos de participantes por periodo escolar, en una parte del artículo dicen que son periodos anuales y más adelante hablan de semestres, luego hablan de que no pudieron tomar datos de los alumnos de últimos semestres que salen a prácticas o clases fuera de la universidad (pero que probablemente sean ellos los que enfrentan más estresores que pueden generar los malestares en salud) y no justifican por qué (no es una justificación suficientemente válida para el diseño, que no están en la universidad).

\section{Resultados}

En la redacción de los resultados, se recomienda especificar con número y porcentaje en algunos de sus párrafos; revisar detenidamente la información de Tablas y Figuras, que no se repita o falte información. Se hace la recomendación de insertar tablas en ciertos párrafos para mejor entendimiento. Dentro de los resultados no se menciona la relación que existe entre ansiedad, depresión y actividad autonómica, la cual es mencionada en el resumen y dentro de la introducción, como objetivo.

Algunas de las tablas que presentan podrían evitarse, por ejemplo la del protocolo de reclutamiento de participantes. Mi evaluación con respecto a los análisis estadísticos es porque me parece que no justificaron por qué hacer todos esos análisis desde su objetivo. Y como comenté previamente, en ocasiones no están considerando el tamaño de sus grupos para elegir sus análisis, por ejemplo en la comparación por sexos. Respecto a las tablas, si hacen correlaciones es apropiado tener la tabla de correlación, si hacen ANOVA, es mejor hacer la tabla ANOVA como sugiere APA reportar ese resultado, tienen algunas tablas que podrían evitarse para incluir estas del análisis. 


\begin{tabular}{|c|c|}
\hline Revisor 1 & Revisor 2 \\
\hline \multicolumn{2}{|c|}{ Discusión } \\
\hline $\begin{array}{l}\text { Se recomienda discutir más sobre los resultados obte- } \\
\text { nidos en cuanto a ansiedad y depresión con datos so- } \\
\text { ciodemográficos, así como resultados de la actividad } \\
\text { autonómica. Si se estudió la relación entre los dos es- } \\
\text { tados emocionales y la actividad autonómica como se } \\
\text { especifica en el resumen e introducción, se debe anexar } \\
\text { resultados y discutir sobre ellos. }\end{array}$ & $\begin{array}{l}\text { En la discusión, sugiero que comiencen explicando si se } \\
\text { afirman o niegan las hipótesis que tenían, también es im- } \\
\text { portante que traten de no especular sobre los trastornos } \\
\text { de ansiedad y depresión, porque no es eso lo que evalua- } \\
\text { ron con sus mediciones y porque los datos que recopila- } \\
\text { ron hablan solamente de niveles moderados de ansiedad y } \\
\text { casi nulos de depresión. Me parece que en la discusión de- } \\
\text { bieran evitar hablar de los sexos diferenciadamente (por } \\
\text { las razones que les expuse previamente), que traten de dar } \\
\text { explicación a por qué los jóvenes de esa universidad no } \\
\text { reportan niveles elevados de ansiedad y depresión, si ge- } \\
\text { neralmente los estudios hablan de ese tipo de problemas, } \\
\text { también será importante que eviten hablar de procesos } \\
\text { de intervención (ej. yoga y terapia cognitivo-conductual) } \\
\text { pues su tesis no perseguía precisamente ese objetivo y apa- } \\
\text { rece descontextualizado de lo que están diciendo. Falta } \\
\text { describir las limitaciones del estudio. }\end{array}$ \\
\hline \multicolumn{2}{|c|}{ Conclusiones } \\
\hline $\begin{array}{l}\text { Mejorar conclusión de acuerdo a las directrices para au- } \\
\text { tores: ¿Cuál es la importancia teórica, clínica o práctica } \\
\text { de los resultados y cuál la base para estas interpretacio- } \\
\text { nes? Si los descubrimientos son válidos y replicables. } \\
\text { ¿Qué fenómenos reales pueden ser explicados o mode- } \\
\text { lados por los resultados? ¿La investigación garantiza las } \\
\text { aplicaciones? ¿Qué problemas siguen sin resolverse o } \\
\text { cuáles surgen a causa de estos descubrimientos? }\end{array}$ & $\begin{array}{l}\text { Sugiero que revisen los criterios que se evalúan en esta } \\
\text { sección para desarrollarla con base en estos y que eso } \\
\text { ayude a completar esta sección. }\end{array}$ \\
\hline \multicolumn{2}{|l|}{$\begin{array}{ll} & \text { Referencias } \\
\end{array}$} \\
\hline No hay observaciones para este apartado. & $\begin{array}{l}\text { En algunas referencias faltó un punto o coma y también } \\
\text { sugiero revisar el manual APA para cuando una refe- } \\
\text { rencia tiene más de } 5 \text { autores. Las siguientes referencias } \\
\text { no aparecen en el texto o tienen un error en el apellido, } \\
\text { revisar: Regehr, C., Glancy, D. \& Pitts, A. (2013) Inter- } \\
\text { ventions to reduce stress in university students: A re- } \\
\text { view and meta-analysis. Journal of Affective Disorders, } \\
\text { 148(1): 1-11. doi: } 10.1016 / \text { j.jad.2012.11.026. Sevinc, } \\
\text { G., Hölzel, B. K., Hashmi, J., Greenberg, J., McCallis- } \\
\text { ter, A., Treadway, ... Lazar, S. W. (2018). Common and } \\
\text { dissociable neural activity after mindfulness-based } \\
\text { stress reduction and relaxation response programs. } \\
\text { Psychosomatic medicine, } 80(5), 439-451 \text {. doi: } 10.1097 / \\
\text { PSY.0000000000000590 }\end{array}$ \\
\hline
\end{tabular}




\section{Historia del Proceso}

\section{EDITORIAL}

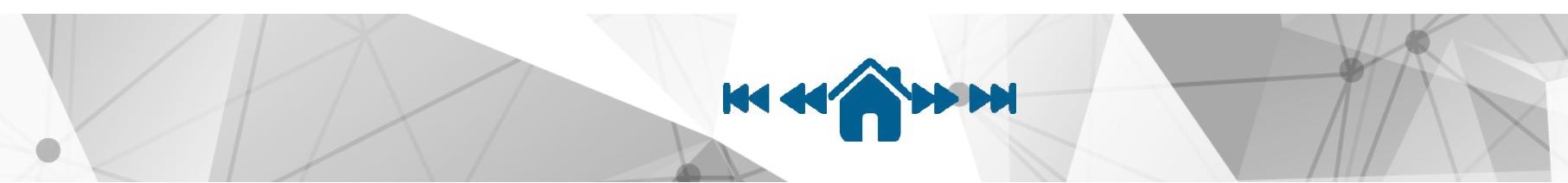

